\title{
Geranylgeranylacetone suppresses colitis-related mouse colon carcinogenesis
}

\author{
TAKUYA INOUE, NAOKI YORIFUJI, MUNETAKA IGUCHI, KAORI FUJIWARA, KAZUKI KAKIMOTO, \\ SADAHARU NOUDA, TOSHIHIKO OKADA, KEN KAWAKAMI, YOSUKE ABE, \\ TOSHIHISA TAKEUCHI and KAZUHIDE HIGUCHI
}

Second Department of Internal Medicine, Osaka Medical College, Takatsuki, Osaka 569-8686, Japan

Received October 23, 2014; Accepted January 12, 2015

DOI: $10.3892 /$ or.2015.3794

\begin{abstract}
Geranylgeranylacetone (GGA), an isoprenoid compound, is an anti-ulcer drug developed in Japan. GGA protects a variety of cells and tissues against numerous stresses via induction of heat shock protein (HSP) 70, and it has recently been reported to protect mice from experimental ulcerative colitis (UC). However, it is unknown whether GGA exhibits a preventive effect on UC-associated neoplasia. In the present study, we evaluated the preventive effects of GGA on colitis-related carcinogenesis in the mouse colon. Mice were administered 1,2-dimethylhydrazine (DMH) subcutaneously three times within a week, followed by 2 cycles of dextran sulfate sodium (DSS) (each cycle, $3 \%$ DSS for 7 days and then distilled water for 14 days) and they were sacrificed 28 days after the completion of the 2 cycles. The mice were divided into the following groups according to the diet received during the experiment: group $\mathrm{A}$, which received a standard diet and served as a disease control; group $\mathrm{B}$, which received a diet mixed with $0.25 \%$ GGA; group $C$, which received a diet mixed with $0.5 \%$ GGA; group $\mathrm{D}$, which received a diet mixed with $1.0 \%$ GGA; group E, which received a diet mixed with $2.0 \%$ GGA; and group F, which received a diet containing no agents, including DSS and served as a normal control. The incidence of neoplasia was assessed. The expression of inducible nitric oxide synthase (iNOS) and 8-hydroxy-2'-deoxyguanosine (8-OHdG) was also determined. In addition, the expression of HSP70 in the colon tissues was determined by immunohistochemistry and western blot analysis. The mean number of tumors was 16.6, 11.0, 9.4, 5.8, 5.4 and 0 in groups A-F, respectively. GGA significantly suppressed the occurrence of neoplasia in a dose-dependent manner. GGA treatment enhanced the expression of HSP70 and suppressed the oxidative damage in the background mucosa (i.e. lesion-free colon).
\end{abstract}

Correspondence to: Dr Takuya Inoue, Second Department of Internal Medicine, Osaka Medical College, 2-7 Daigakumachi, Takatsuki, Osaka 569-8686, Japan

E-mail: ureuretakuwan@yahoo.co.jp

Key words: geranylgeranylacetone, dextran sulfate sodium, cancer, HSP70
These results suggest that GGA could be useful in the prevention of UC-associated neoplasia.

\section{Introduction}

Colorectal cancer is one of the most serious complications of ulcerative colitis (UC), and the risk of UC-associated neoplasia increases as the size of the affected region and the duration of the disease increase. The incidence of UC-associated dysplasia and cancer is higher than that of sporadic colorectal cancer. UC-associated cancer usually occurs at a younger age, has a higher proportion of multiple lesions and shows a poorer survival rate than sporadic colorectal cancer (1). 5-Aminosalicylates (5-ASAs) are widely used to treat patients with UC, and experimental data suggest that 5-ASAs reduce the risk of UC-associated dysplasia and cancer; however, observational studies investigating the effect of 5-ASAs on UC-associated dysplasia and cancer have revealed conflicting results (2-4). Therefore, the need for more effective chemoprevention of UC-associated dysplasia and cancer is well acknowledged (5).

Heat shock protein (HSP) 70 is a stress-inducible protein with a strong cytoprotective effect against numerous stresses (6). HSP induction inhibited inducible nitric oxide synthase (iNOS), which is related to oxidative stress and subsequent transversion mutation of DNA, and prevented dysplastic lesions in an inflammatory model of colon cancer (7-9). Geranylgeranylacetone (GGA), an isoprenoid compound, is an anti-ulcer drug developed in Japan. GGA has been demonstrated to protect a variety of cells and tissues from damage via induction of HSP70 (6). It has recently been reported that GGA protects mice from experimental colitis, such as dextran sulfate sodium (DSS)-induced colitis and trinitrobenzene sulfonic acid (TNBS)-induced colitis $(10,11)$. However, it is unknown whether GGA exhibits a preventive effect on UC-associated neoplasia. The present study investigated the preventive effects of GGA on colitis-related mouse colon carcinogenesis.

\section{Materials and methods}

Animals. Six-week-old female BALB/c mice (Clea Japan, Tokyo, Japan) weighing 20-25 g were used in the present 
study. The animals were maintained in an animal colony with controlled temperature $\left(23^{\circ} \mathrm{C}\right)$ and light $(12 / 12$-h light and dark cycle) at the Osaka Medical College (OMC; Osaka, Japan), and were permitted free access to standard mouse chow pellets (MM-3; Funabashi, Chiba, Japan) and tap water.

Protocol for induction of colorectal tumors and experimental procedures. Colorectal tumors and experimental colitis were induced as previously described (12). In brief, the mice received 1,2-dimethylhydrazine (DMH; Wako Pure Chemical Industries, Osaka, Japan) at a dose of $20 \mathrm{mg} / \mathrm{kg}$ body weight subcutaneously three times within a week. Starting 1 week after the DMH injection, chronic colitis was induced by the administration of 2 cycles of DSS (molecular weight, 5,000; Meitou Sangyou, Osaka, Japan) (each cycle, 3\% DSS for 7 days and then distilled water for 14 days). The mice were sacrificed 28 days after the completion of the 2 cycles.

The mice were divided into six groups (A-F) according to the diet they received during the experiment. Group A received a standard diet and served as a disease control; group B received a diet mixed with $0.25 \%$ of GGA (kindly provided by Eisai Co., Tokyo, Japan); group $\mathrm{C}$ received a diet mixed with $0.5 \%$ GGA; group $\mathrm{D}$ received a diet mixed with 1.0\% GGA; group E received a diet mixed with $2.0 \%$ GGA; group $\mathrm{F}$ received a diet containing no agents, including DSS and served as a normal control.

The entire colorectum from the colocecal junction to the anal verge was excised and rinsed in phosphate-buffered saline (PBS). The specimen was opened longitudinally and was fixed on a cork board in $10 \%$ formalin. Then, the specimen was stained with $0.2 \%$ methylene blue, and the number of colonic tumors was counted under a stereomicroscope $(13,14)$. Histopathological examination was performed on paraffin-embedded sections after hematoxylin and eosin staining. Colonic mucosal dysplasia and cancer were diagnosed according to the criteria described by Ridell et al (15).

Evaluation of the severity of clinical colitis. The disease activity index (DAI) was determined in all the animals during the first DSS administration cycle by scoring the body weight, the stool hemoccult reactivity or the presence of gross blood and stool consistency, in accordance with the method described by Murthy et al (16). This method of scoring is a comprehensive functional measure that correlates well with the degree of inflammation. The individuals who examined the mice and determined the DAIs were blinded as to the experimental group to which the animal belonged.

Immunohistochemistry and immunoblot analyses. Expression of HSP70 and 8-hydroxy-2'-deoxyguanosine (8-OHdG) in the intestinal mucosa was assessed by the labeled streptavidinbiotin method with the LSAB kit (Dako, Carpinteria, CA, USA) with microwave accentuation. Each segment was fixed in $10 \%$ formalin, embedded in paraffin wax and cut into tissue sections of 4-mm thickness. Tissue sections were mounted on microscope slides, deparaffinized in xylene $(3 \times 3 \mathrm{~min})$ and dehydrated with $100 \%$ ethanol. After washes with PBS, sections were placed in $10 \mathrm{mmol} / \mathrm{l}$ citrate buffer $(\mathrm{pH} 6.0)$ and heated to $80^{\circ} \mathrm{C}$ for $10 \mathrm{~min}$ in a microwave oven. After washes with PBS, endogenous peroxidase activity was blocked with $0.3 \%$ hydrogen peroxide in $10 \%$ methanol for $30 \mathrm{~min}$, and blocking reagent was added for $15 \mathrm{~min}$. The sections were incubated at $4^{\circ} \mathrm{C}$ overnight in the primary antibody (anti-HSP70 IgG; Stressgen, Ann Arbor, MI, USA) (anti-8-OHdG IgG; NOF Co., Tokyo, Japan). After washes with PBS, the sections were incubated with a biotinylated immunoglobulin antibody (Dako) at room temperature for $30 \mathrm{~min}$. The sections were then washed in PBS and visualized with streptavidin-biotin horseradish peroxidase and 3,3'-diaminobenzidine (both from Dako). Finally, the sections were counterstained with hematoxylin, dehydrated and coverslipped with permanent mounting medium for examination under a light microscope (13).

Lysates of colonic mucosa containing $20 \mu \mathrm{g} / 20 \mu \mathrm{l}$ of protein were separated by electrophoresis through a NuPAGE ${ }^{\circledR} 4-12 \%$ Bis-Tris gel (1.0 mm) (Life Technologies, Carlsbad, CA, USA), and transferred onto polyvinylidene difluoride membranes (Pall Corporation, Port Washington, NY, USA). Membranes were incubated in 5\% skim milk in Tris-buffered saline with Tween-20 (TBST) at room temperature for $10 \mathrm{~min}$ and stored at $4^{\circ} \mathrm{C}$ overnight. After 3 washes in TBST, the membranes were incubated with the mouse anti-HSP70 antibody and mouse anti- $\beta$-actin antibody (Sigma Chemicals, St. Louis, MO, USA) for $1 \mathrm{~h}$ at room temperature in $5 \%$ skim milk. Membranes were washed 3 times in TBST and incubated with peroxidase-conjugated secondary antibodies (Santa Cruz Biotechnology, Dallas, TX, USA). Blots were washed 3 times with TBST and developed with an enhanced chemiluminescence system (ECL Prime Western Blotting Detection System; GE Healthcare, Buckinghamshire, UK) and Fujifilm Imaging System Application Note LAS-3000 (Fujifilm, Tokyo, Japan).

Analysis of iNOS mRNA expression by reverse transcription $P C R$. To evaluate iNOS mRNA expression in background mucosa, a small sample of intestinal tissue was removed from the lesion-free mouse colon under a stereomicroscope, frozen in liquid nitrogen, and stored at $-80^{\circ} \mathrm{C}$ until RNA isolation. Total RNA was extracted from tissue samples with the total RNEasy Mini kit (Qiagen GmbH, Hilden, Germany). Reverse transcription and polymerase chain reaction were performed with the high-fidelity prime script RT-PCR kit (Takara Bio, Inc., Shiga, Japan) according to the manufacturer's instructions. The sequences of sense and antisense primers for the mouse iNOS were, 5'-TGTCAGTGGCTTCCAGCTCC-3' and 5'-TAGTCTT CCACCTGCTCCTC-3', giving rise to a 450-bp PCR product. For mouse glyceraldehyde-3-phosphate dehydrogenase (G3DPH), a constitutively expressed gene, the sequence was, 5'-TGAAGGTCGGTGTGAACGGATTTGC-3' for the sense primerand5'-CATGTAGGCCATGAGGTCCACC AC-3' for the antisense primer, giving rise to a 983-bp PCR product. An aliquot of the reverse transcription reaction product for iNOS expression served as a template in 35 cycles of PCR with $10 \mathrm{sec}$ of denaturation at $98^{\circ} \mathrm{C}, 5 \mathrm{sec}$ of annealing at $55^{\circ} \mathrm{C}$ and $0.5 \mathrm{~min}$ of extension at $72^{\circ} \mathrm{C}$ on the thermal cycler. A portion of the PCR mixture was electrophoresed on $1.5 \%$ agarose gel in Tris-EDTA-acetic acid buffer, and the gel was stained with ethidium bromide and photographed.

Statistical analysis. All the results are expressed as means \pm SD. Comparisons were carried out with a one-way analysis of variance (ANOVA) or the Kruskal-Wallis test 


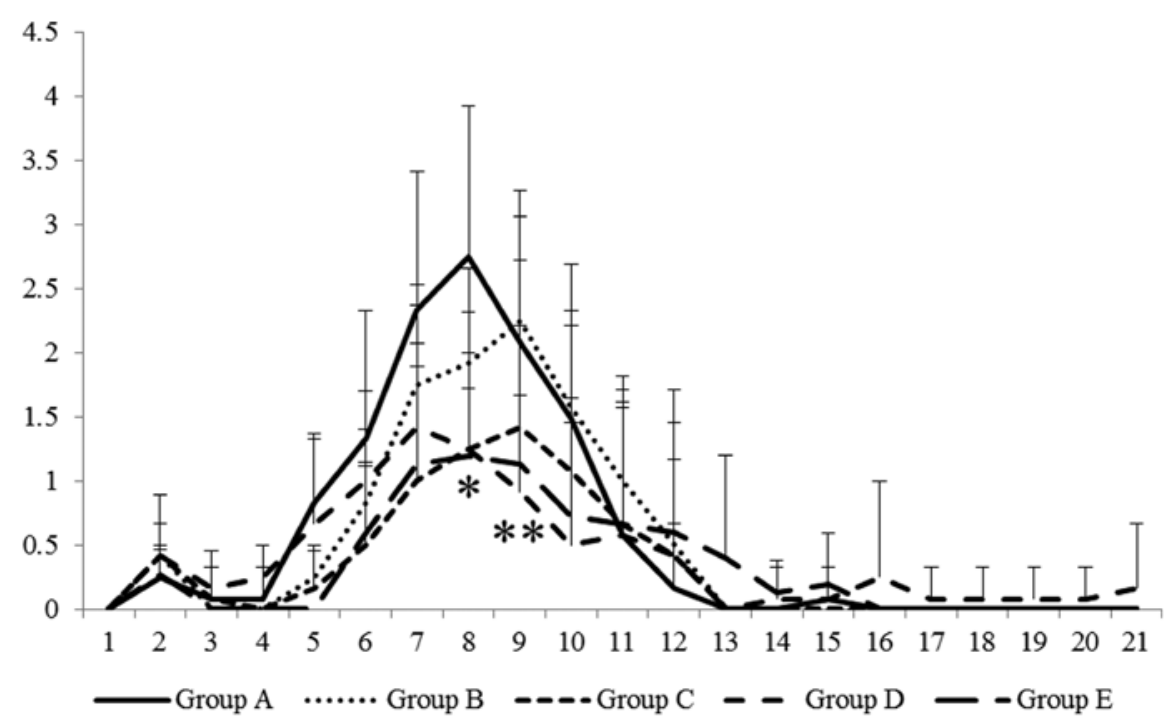

Figure 1. Time course of the DAI after the initiation of DSS administration. " $p<0.05$ groups C-E compared to group A on day 8 . ${ }^{* *} p<0.05$ group D compared to group A on day 9. DAI, disease activity index.

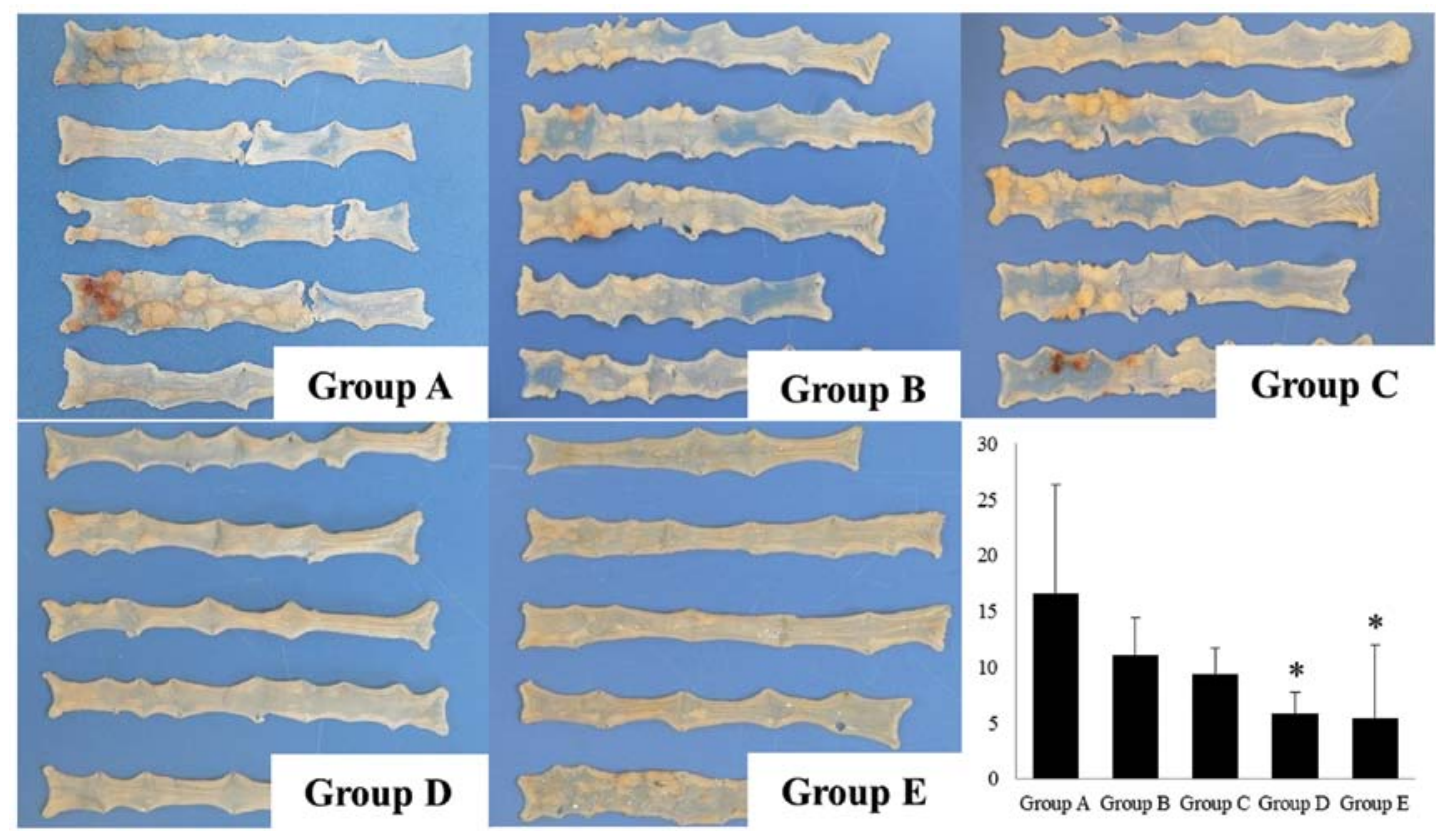

Figure 2. Colorectal tumors in mice. Macroscopic view showing gross lesions in the colon. GGA dose-dependently suppressed the occurrence of colon tumorigenesis. " $\mathrm{p}<0.05$ compared to group A. GGA, geranylgeranylacetone.

followed by Fisher's PLSD test. Statistical significance was defined as $\mathrm{p}<0.05$.

\section{Results}

Changes in DAI. As previously described (12), the DAI gradually increased until day 8 , and usually reverted to normal by day 21. Clinical symptoms of colitis, including bloody stool, diarrhea and loss of body weight, progressed until day 8 . Although there was no significant difference between the groups, body weight loss was dose-dependently attenuated by GGA treatment. Accordingly, the administration of GGA significantly suppressed the DAI in a dose-dependent manner (Fig. 1).
Colorectal length and number of tumors. The colorectal length in groups A-F was $8.3 \pm 0.4,8.2 \pm 0.4,8.7 \pm 1.1,9.7 \pm 0.2$, $9.4 \pm 0.7$ and $9.6 \pm 0.3 \mathrm{~cm}$, respectively. The colorectal length was significantly shortened in groups A-E compared to the normal control (group F) 70 days after the start of DSS administration. The administration of GGA significantly reduced the decrease in colon length (groups D and E) compared to the disease control (group A). Although the incidence of colonic neoplasia (number of mice with neoplasms) in groups A-E was $100 \%$, the multiplicity (number of tumors/mouse) in groups A-E was 16.6 $\pm 9.7,11.0 \pm 3.4,9.4 \pm 2.3,5.8 \pm 1.9$ and $5.4 \pm 6.6$, respectively (Fig. 2). The administration of GGA significantly suppressed the number of tumors/mouse in a dose-dependent manner (Fig. 2). 

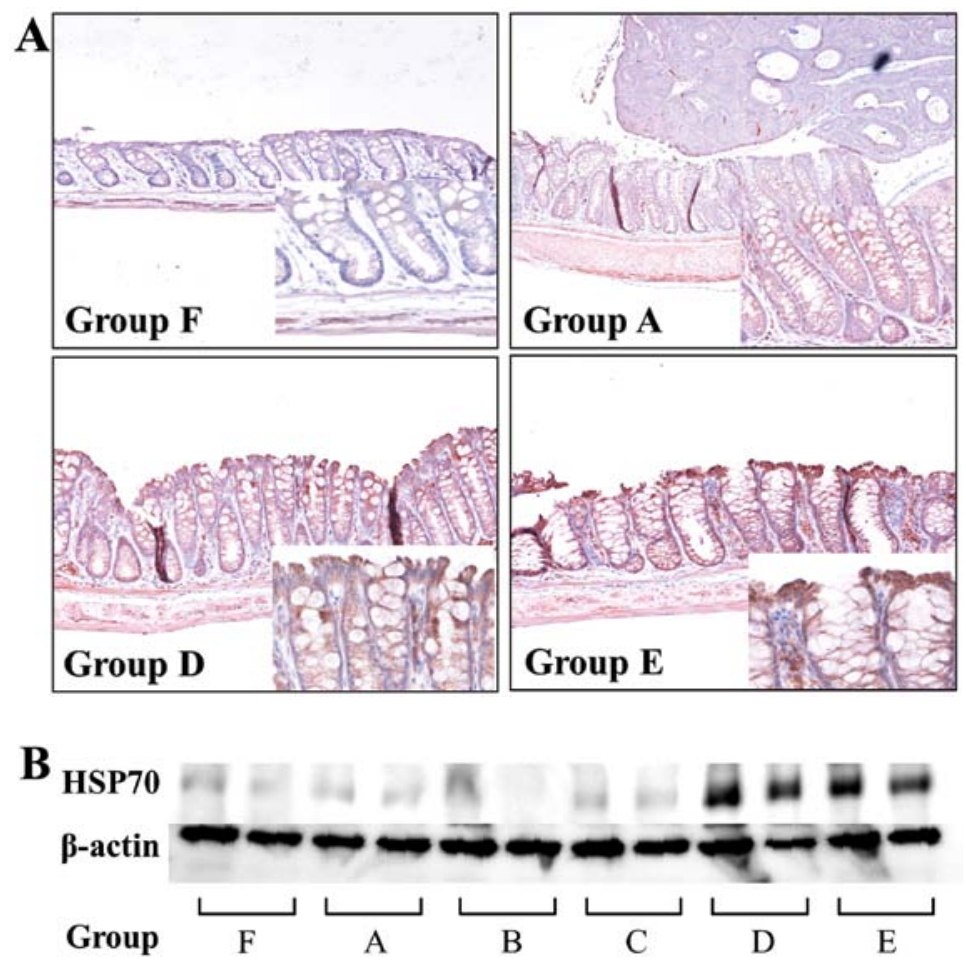

Figure 3. HSP70 expression in the background mucosa by (A) immunohistochemistry and (B) immunoblotting. The administration of GGA enhanced the cytoplasmic HSP70 expression in the epithelium of the background mucosa. Western blot analysis also showed that mucosal HSP70 expression was increased in the GGA-treated mice (groups D and E). GGA, geranylgeranylacetone.
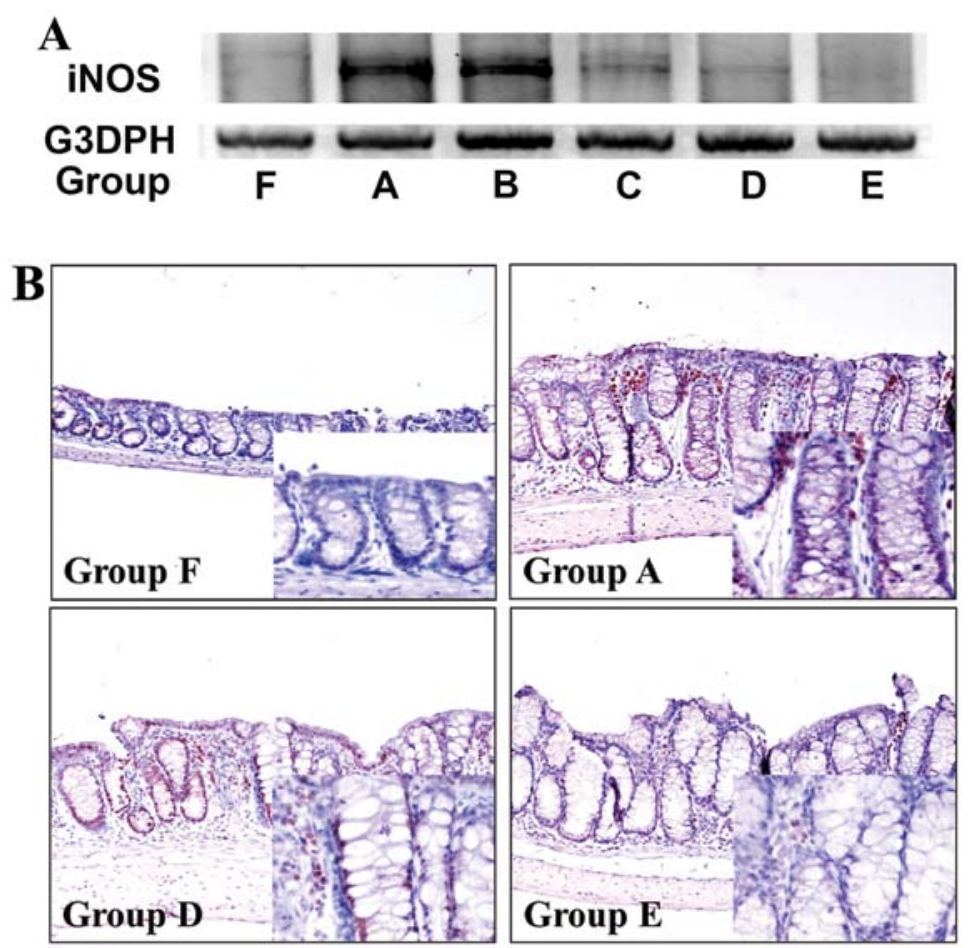

Figure 4. iNOS and 8-OHdG expression in the background mucosa by (A) RT-PCR and (B) immunohistochemistry. iNOS mRNA expression, which was upregulated in DSS-treated mouse colons, was decreased by the administration of GGA. GGA also markedly decreased the accumulation of 8-OHdG, which was strongly expressed in the background mucosa of group A, suggesting that GGA suppressed colitis-induced mouse colon carcinogenesis via suppression of oxidative DNA damage. iNOS, inducible nitric oxide synthase; 8-OHdG, 8-hydroxy-2'-deoxyguanosine; GGA, geranylgeranylacetone.

HSP70 expression. Since it has been reported that GGA induces HSP70, which has a protective effect against inflammation, and the DAI was significantly suppressed by GGA treatment in the present study, we examined the expression of HSP70 in the background mucosa (i.e. lesion-free colon) by immunohistochemistry and immunoblotting. As shown 
in Fig. 3A, immunohistochemistry revealed weak positivity for HSP70 in the colon of the normal (group F) and disease control (group A) mice. The administration of GGA enhanced the cytoplasmic HSP70 expression in the epithelium of the background mucosa. Western blot analysis also showed that mucosal HSP70 expression was increased in the GGA-treated mice (groups D and E) (Fig. 3B).

iNOS and 8-OHdG expression. It is well known that iNOS can cause oxidative stress and leads to $8-\mathrm{OHdG}$ accumulation. Since GGA has been reported to increase mucosal HSP70 without increasing the $8-\mathrm{OHdG}$ production, we analyzed iNOS and 8-OHdG expression by reverse-transcription PCR and immunohistochemistry. As shown in Fig. 4A, iNOS mRNA expression in the background mucosa was upregulated in groups A and B. However, iNOS mRNA expression was decreased in groups $\mathrm{C}$-E. Immunohistochemistry revealed that 8-OHdG was strongly expressed in the background mucosa of group A, and the administration of GGA resulted in markedly decreased 8-OHdG expression (Fig. 4B). These results suggest that GGA suppressed colitis-induced mouse colon carcinogenesis via suppression of oxidative DNA damage.

\section{Discussion}

It is well known that HSPs are induced by various stressors and protect cells from such stressors (6). In particular, HSP70 has been reported to provide protective effects against gastrointestinal diseases, such as gastric ulcer, experimental colitis, and nonsteroidal anti-inflammatory drug (NSAID)-induced enteritis in rodents $(10,11,17,18)$. Regarding NSAID-induced intestinal mucosal injuries, we recently demonstrated via video capsule endoscopy that GGA markedly inhibited the development of lesions in human subjects (19). Since GGA increases mucosal HSP70 expression without increasing the oxidative damage followed by $8-\mathrm{OHdG}$ accumulation (20), GGA is expected to be therapeutically beneficial for gastrointestinal tract diseases as a non-toxic HSP inducer. However, to date, it remains unknown whether GGA exhibits a preventive effect in UC-associated neoplasia. To our knowledge, the present study demonstrated for the first time that the administration of GGA exerts a suppressive effect on the development of neoplasia in a murine model of colitis.

Cyclooxygenase (COX)-2, which is an inducible COX and upregulated in response to various stimuli, such as interleukin-1 and tumor necrosis factor, is progressively overexpressed during the stepwise sequence from adenoma to cancer (21). Therefore, NSAIDs are often administered to patients with polyposis to inhibit COX activity and induce apoptosis, which suppresses 8-OHdG formation (22). However, for patients with $\mathrm{UC}$, it is thought that NSAIDs may trigger UC relapse, so they should not be administered to patients with UC (23). Indeed, we previously administered a COX-2 inhibitor to mice with DSS-induced colitis and demonstrated that the presence of a COX-2 inhibitor during colitis exacerbated the colitis and did not show a preventive effect against colitis-induced tumorigenesis (13). Nishida et al evaluated the protective effect of GGA against noxious agents in rat gastric mucosa and demonstrated that GGA dose-dependently increased COX-2 expression and prostaglandin E2 production (24). In the present study, the administration of GGA suppressed iNOS expression and dose-dependently attenuated DSS-induced colitis. Therefore, we consider that these characteristics of GGA may be suitable for patients with UC to prevent colitis-associated cancer development.

According to the National Center for Advancing Translational Sciences, the term 'drug repurposing' generally refers to the study of a compound or biologic to treat one disease or condition to see whether it is safe and effective for treating other diseases (http://www.ncats.nih.gov/). Since developing a new drug takes an enormous amount of time, and repurposing (repositioning) candidates have often been through several stages of clinical development and have well-known safety and pharmacokinetic profiles, drug repurposing (repositioning) may enable a faster development time and reduce risks (25). Since it is well known that GGA has no significant adverse effects and is an inexpensive drug, GGA is considered to be one of the most suitable agents for drug repurposing (repositioning), and its use is being attempted in other models of disease in Japan $(26,27)$.

In conclusion, although it remains unknown whether the usual dose for patients with gastric ulcer will be approved for patients with UC, the results of the present study suggest that GGA could be a useful therapeutic agent for the prevention of UC-associated cancer. Further studies to evaluate the effect of GGA on the human colonic mucosa and to clarify the optimal dose for colonic inflammation are required.

\section{References}

1. Watanabe T, Konishi T, Kishimoto J, Kotake K, Muto T, Sugihara K; Japanese Society for Cancer of the Colon and Rectum: Ulcerative colitis-associated colorectal cancer shows a poorer survival than sporadic colorectal cancer: a nationwide Japanese study. Inflamm Bowel Dis 3: 802-808, 2011.

2. Lyakhovich A and Gasche C: Systematic review: molecular chemoprevention of colorectal malignancy by mesalazine. Aliment Pharmacol Ther 31: 202-209, 2010.

3. Herfarth $\mathrm{H}$ : The role of chemoprevention of colorectal cancer with 5-aminosalicylates in ulcerative colitis. Dig Dis 30 (Suppl 2): 55-59, 2012

4. Lopez A and Peyrin-Biroulet L: 5-Aminosalicylic acid and chemoprevention: does it work? Dig Dis 31: 248-253, 2013.

5. Ekbom A, Helmick C, Zack M and Adami HO: Ulcerative colitis and colorectal cancer. A population-based study. N Engl J Med 323: 1228-1233, 1990.

6. Mizushima T: RETRACTED: protective role of HSP70 against various gastrointestinal diseases. Curr Opin Pharmacol 19: 1-5, 2014.

7. Hauser GJ, Dayao EK, Wasserloos K, Pitt BR and Wong HR: HSP induction inhibits iNOS mRNA expression and attenuates hypotension in endotoxin-challenged rats. Am J Physiol 271: H2529-H2535, 1996.

8. Chan JY, Ou CC, Wang LL and Chan SH: Heat shock protein 70 confers cardiovascular protection during endotoxemia via inhibition of nuclear factor- $\kappa \mathrm{B}$ activation and inducible nitric oxide synthase expression in the rostral ventrolateral medulla. Circulation 110: 3560-3566, 2004.

9. Tao Y, Hart J, Lichtenstein L, Joseph LJ, Ciancio MJ, Hu S, Chang EB and Bissonnette M: Inducible heat shock protein 70 prevents multifocal flat dysplastic lesions and invasive tumors in an inflammatory model of colon cancer. Carcinogenesis 30: 175-182, 2009.

10. Ohkawara T, Nishihira J, Takeda H, Miyashita K, Kato K, Kato M, Sugiyama T and Asaka M: Geranylgeranylacetone protects mice from dextran sulfate sodium-induced colitis. Scand J Gastroenterol 40: 1049-1057, 2005.

11. Ohkawara T, Nishihira J, Takeda H, Katsurada T, Kato K, Yoshiki T, Sugiyama T and Asaka M: Protective effect of geranylgeranylacetone on trinitrobenzene sulfonic acid-induced colitis in mice. Int J Mol Med 17: 229-234, 2006. 
12. Inoue T, Murano M, Yoda Y, et al: R-etodolac induces E-cadherin and suppresses colitis-related mouse colon tumorigenesis. Oncol Rep 24: 1487-1492, 2010.

13. Inoue T, Murano M, Abe Y, et al: Therapeutic effect of nimesulide on colorectal carcinogenesis in experimental murine ulcerative colitis. J Gastroenterol Hepatol 22: 1474-1481, 2007.

14. Wedemeyer J and Galli SJ: Decreased susceptibility of mast cell-deficient $\mathrm{Kit}^{W} / \mathrm{Kit}^{W-v}$ mice to the development of 1,2-dimethylhydrazine-induced intestinal tumors. Lab Invest 85: 388-396, 2005.

15. Riddell RH, Goldman H, Ransohof DF, et al: Dysplasia in inflammatory bowel disease: standardized classification with provisional clinical application. Human Pathol 14: 931-968, 1983.

16. Murthy SN, Cooper HS, Shim H, Shah RS, Ibrahim SA and Sedergran DJ: Treatment of dextran sulfate sodium-induced murine colitis by intracolonic cyclosporine. Dig Dis Sci 38: 1722-1734, 1993.

17. Suemasu S, Tanaka K, Namba T, et al: A role for HSP70 in protecting against indomethacin-induced gastric lesions. J Biol Chem 284: 19705-19715, 2009.

18. Asano T, Tanaka K, Yamakawa N, Adachi H, Sobue G, Goto H, Takeuchi K and Mizushima T: HSP70 confers protection against indomethacin-induced lesions of the small intestine. J Pharmacol Exp Ther 330: 458-467, 2009.

19. Umegaki E,Kuramoto T,Kojima Y,Nouda S, Ishida K, Takeuchi $T$, Inoue T, Tokioka S and Higuchi K: Geranylgeranylacetone, a gastromucoprotective drug, protects against NSAID-induced esophageal, gastroduodenal and small intestinal mucosal injury in healthy subjects: a prospective randomized study involving a comparison with famotidine. Intern Med 53: 283-290, 2014.
20. Yanaka A, Zhang S, Sato D, Tauchi M, Suzuki H, Shibahara T, Matsui H, Nakahara A and Hyodo I: Geranylgeranylacetone protects the human gastric mucosa from diclofenac-induced injury via induction of heat shock protein 70. Digestion 75: 148-155, 2007.

21. Chan AT, Ogino S and Fuchs CS: Aspirin and the risk of colorectal cancer in relation to the expression of COX-2. N Eng J Med 356: 2131-2142, 2007.

22. Tardieu D, Jaeg JP, Deloly A, Corpet DE, Cadet J and Petit CR: The COX-2 inhibitor nimesulide suppresses superoxide and 8-hydroxy-deoxyguanosine formation, and stimulates apoptosis in mucosa during early colonic inflammation in rats. Carcinogenesis 21: 973-976, 2000.

23. Forrest K, Symmons D and Foster P: Systematic review: is ingestion of paracetamol or non-steroidal anti-inflammatory drugs associated with exacerbations of inflammatory bowel disease? Aliment Pharmacol Ther 20: 1035-1043, 2004

24. Nishida T, Yabe Y, Fu HY, Hayashi Y, Asahi K, Eguchi H, Tsuji S, Tsujii M, Hayashi N and Kawano S: Geranylgeranylacetone induces cyclooxygenase-2 expression in cultured rat gastric epithelial cells through NF- $\kappa$ B. Dig Dis Sci 52: 1890-1896, 2007.

25. Ashburn TT and Thor KB: Drug repositioning: identifying and developing new uses for existing drugs. Nat Rev Drug Discov 3: 673-683, 2004

26. Hoshino T, Suzuki K, Matsushima T, Yamakawa N, Suzuki T and Mizushima T: Suppression of Alzheimer's disease-related phenotypes by geranylgeranylacetone in mice. PLoS One 8: e76306, 2013.

27. Namba T, Tanaka K, Hoshino T, Azuma A and Mizushima T: Suppression of expression of heat shock protein 70 by gefitinib and its contribution to pulmonary fibrosis. PLoS One 6: e27296, 2011. 